\title{
Tuberculosis en el personal de salud
}

\author{
Comité Consultivo de Infecciones Intrahospitalarias Sociedad Chilena de Infectología \\ Alberto Fica C., Marcela Cifuentes D., M. Cristina Ajenjo H., M. Irene Jemenao P., Alejandra Zambrano G., \\ Naldy Febré V., Luis Delpiano M., Alexis Diomedi P. y Paulina Ramonda C.
}

\section{Tuberculosis in healthcare workers}

Tuberculosis (TB) is an occupational risk hazard that explains 5 to 5.361 additional cases of TB per 100.000 individuals among healthcare workers $(\mathrm{HCW})$ in relation to general population in developing countries. For each clinical case a number of additional infections are occurring, that can be detected by tuberculin skin test conversion among non-BCG vaccinated $\mathrm{HCW}$ or by interferon-gamma testing. Risk factors for $\mathrm{HCW}$ infection include number of TB patients examined, job characteristics and place of work, delay in diagnostic suspicion, patients with multidrug resistant strains, limited access to appropriate ventilation systems, noncompliance with aerosol dissemination precautions, immune suppressed and/or malnourished HCW. Molecular studies suggest that only 32 to $42 \%$ of TB cases among HCW are related to occupational exposure. Useful measures to prevent occupational TB acquisition include a number of administrative-, infrastructure- and personal-related measures that have proven to be successful in reducing occurrence of new infections including clinical TB cases among $\mathrm{HCW}$. In Chile, two official government sponsored guidelines are currently available for preventing TB infection among HCW, issued by the national TBC Control Program and by the National Nosocomial infection Control Program. Major differences in recommendations between these guidelines indicate that an update is urgently needed.

Key words: Tuberculosis, epidemiology, healthcare workers, risk factors, prevention.

Palabras clave: Tuberculosis, epidemiología, personal de salud, factores de riesgo, prevención.

\section{Introducción}

$\mathrm{E}$ 1 riesgo de tuberculosis (TBC) nosocomial fue un hecho bien conocido en el pasado, que evolucionó hacia una aparente tranquilidad en la segunda mitad del siglo XX debido al advenimiento de una terapia específica y a la declinación global de esta enfermedad en el mundo. Sin embargo, esta relación pacífica se quebró antes del término del mismo siglo, debido a la re-emergencia de esta condición mórbida y a la aparición sustantiva de casos asociados a cepas multi-resistentes que se asociaron a una mayor letalidad. En este fenómeno, los casos nosocomiales, ya sea por transmisión hacia otros pacientes o hacia el propio personal de salud (PS), volvieron a captar la atención mundial.

El Comité Consultivo de IIH de la Sociedad Chilena de Infectología, en conjunto con el Programa de TBC del Servicio de Salud Metropolitano Sur de Santiago, decidieron efectuar una revisión del tema, abarcando diferentes aspectos y que contemplan: fundamentos del riesgo, su magnitud y factores asociados, incluyendo información nacional; el diagnóstico de infección latente en el PS, especialmente en población con antecedentes de vacunación $\mathrm{BCG}$; las medidas de preven- ción, su organización y eficacia y, finalmente, la situación actual de las medidas de prevención en Chile.

El Comité Consultivo piensa que esta revisión es necesaria en nuestro medio debido a la reciente aparición de una norma técnica revisada sobre el Control de la TBC en Chile y a la necesidad de establecer un debate informado sobre la materia. Complementa además, una revisión previa sobre el tema que data de hace 10 años ${ }^{1}$. Este documento está organizado en la secuencia antes señalada.

Fundamentos, magnitud del riesgo y factores asociados. El agente de la TBC es eliminado por pacientes con TBC pulmonar bacilífera hacia el ambiente, a través de diferentes acciones como la tos, el estornudo, el acto de escupir, cantar o incluso la conversación ${ }^{2}$. Las gotas que se generan se evaporan rápidamente y se convierten en aerosoles de pequeñas partículas que, por su tamaño (1 a $3 \mu \mathrm{m})$, permanecen en suspensión y pueden ser transportadas, según el flujo del aire, a través de la habitación o de un edifício. El pequeño tamaño facilita su llegada a los alvéolos pulmonares y el riesgo de transmisión está confinado a los que comparten lugares cerrados y mal ventilados, debido a la gran concentración de aerosoles en ese espacio.

\author{
Hospital Clínico Universidad de \\ Chile \\ Sección Infectología (AFC) y Comité \\ de IIH (AFC, MIJP) \\ Complejo Hospitalario San \\ Borja Arriarán \\ Comité de IIH (MCD, LDM) \\ Hospital Clínico Pontificia \\ Universidad Católica de Chile \\ (MCAH) \\ Clínica Las Condes, Santiago \\ Comité de IIH (NFV) \\ Instituto Nacional del Cáncer \\ (ADP) \\ Ministerio de Salud, Chile \\ Servicio de Salud Metropolita- \\ no Sur de Santiago \\ Programa de Tuberculosis (PRC) \\ Recibido: 6 mayo 2008 \\ Aceptado: 13 mayo 2008
}

Correspondencia a:

Alberto Fica Cubillos afica@redclinicauchile.cl 
La comprobación definitiva de la transmisión de la TBC por aerosoles provino de estudios experimentales con cobayos puestos en los techos de las salas de pacientes con TBC pulmonar y de un brote de infección en los tripulantes de un barco de la marina estadounidense detectándose casos en los marinos expuestos al mismo sistema de ventilación, sin contacto directo con el paciente fuente ${ }^{2}$.

\section{Tabla 1. Contagiosidad estimada de la TBC en diferentes circunstancias}

\begin{tabular}{lccc|} 
Condición & $\begin{array}{c}\text { Quanta liberada } \\
\text { por hora }\end{array}$ & $\begin{array}{c}\text { Volumen de aire } \\
\text { que contiene } \\
\text { una quanta }\end{array}$ & $\begin{array}{c}\text { Tiempo laboral } \\
\text { requerido para un } \\
\text { caso de contagio }\end{array}$ \\
$\begin{array}{l}\text { TBC pulmonar cavitada } \\
\text { en sitio con mala } \\
\text { ventilación }\end{array}$ & 13 & No disponible & No disponible \\
\hline $\begin{array}{l}\text { TBC laríngea } \\
\text { Broncoscopia }\end{array}$ & 60 & 5.663 litros & 15 horas \\
\hline $\begin{array}{l}\text { Autopsia } \\
\text { TBC en tratamiento } \\
\text { reciente }\end{array}$ & No disponible & 1.953 litros & 5,4 horas \\
\hline Sarampión & 1,25 & 311.487 litros & 0,5 horas \\
\hline
\end{tabular}

Tabla 2. Factores asociados a un mayor riesgo de infección o de TBC activa en el personal de salud

\section{Condición}

Volumen de pacientes con

TBC atendidos en una institución

Ocupación del PS

Lugar de trabajo del PS

\section{Oportunidad diagnóstica}

Multiresistencia a fármacos anti tuberculosos

Sistemas de ventilación

\section{Comentarios}

El riesgo se incrementa con el número de pacientes anuales atendidos

El riesgo es mayor en enfermeras, terapeutas respiratorios, residentes, estudiantes de pregrado, personal que trabaja en autopsias y en fibrobroncoscopia

EI PS que labora en el área clínica, en microbiología o en autopsias tiene más riesgo

El retraso diagnóstico mantiene el riesgo

En caso de multi-resistencia el paciente mantiene su condición bacilífera

El contagio aumenta en lugares oscuros y mal ventilados, en salas de broncoscopia o de terapia respiratoria que no están adaptados estructuralmente

Medidas de aislamiento por aerosoles

La hospitalización en sala compartida permite el contagio hacia otros pacientes, algunos de ellos inmunocomprometidos

Barreras protectoras para el PS Las máscaras N95 disminuyen el riesgo de contagio si son utilizadas apropiadamente

Inmunosupresión en el PS

La existencia de PS con infección por VIH los expone a infección, progresión a TBC clínica y muerte

Desnutrición en el PS Un índice de masa corporal $<19 \mathrm{~kg} / \mathrm{m}^{2}$ aumenta el riesgo de $T B C$ activa
La transmisión de la TBC hacia el PS y el riesgo de infección, dependen de numerosos factores que explican el amplio rango de frecuencia reportada. Debe tenerse presente que infección no es equivalente a enfermedad, siendo un fenómeno mucho más frecuente la infección que la enfermedad; por cada caso de enfermedad en el PS se han producido muchos casos de infección latente. Luego de la exposición, sólo 5\% de los infectados desarrollará enfermedad en los dos años siguientes y otro $5 \%$ adicional en los años venideros. En forma habitual, la infección por TBC se diagnostica por el viraje de la prueba de tuberculina (de negativa a positiva) durante el seguimiento de una población (PS) con una prueba inicial negativa.

El riesgo de diseminación de la TBC desde un paciente varía si el paciente tiene cavidades pulmonares, tiene TBC laríngea, es sometido a una broncoscopia o está en los primeros días de tratamiento. La dosis infectante o quanta liberada al espacio aéreo (dosis necesaria para infectar a una persona), difiere entre estas condiciones y los expuestos requerirán mayor o menor tiempo de exposición. Si la quanta liberada es elevada se necesitará un menor tiempo de permanencia en una sala donde está el paciente ya que en un menor número de ciclos respiratorios se lograra el contagio $^{3}$. En la Tabla 1 se ilustran estas diferencias. A manera de comparación con la transmisión de otras enfermedades contagiosas, la contagiosidad del sarampión es mucho más elevada.

Magnitud del riesgo. En la era pre-tratamiento antituberculoso, el riesgo estimado de infección anual alcanzaba al $80 \%$ del personal. En la era post-tratamiento anti-tuberculoso y durante la existencia de brotes, este riesgo oscila entre 14 y $55 \%$ para infección latente y entre 2,2 y $8,4 \%$ para TBC clínica ${ }^{3}$. En una extensa revisión para países en vías de desarrollo se recopiló un margen entre 25 y 5.361 casos adicionales de TBC clínica cada 100.000 personas s. $^{4}$

Entre los factores que explican las diferencias en el riesgo de infección o desarrollo de TBC activa destacan: el volumen de pacientes con TBC atendidos, la función u ocupación del PS, el lugar de trabajo del PS, el retraso en el diagnóstico de los pacientes con TBC, diferencias en ventilación ambiental, la aplicación o no aplicación de medidas de aislamiento para aerosoles, las barreras de protección que usa el personal clínico y la existencia de PS con alguna condición de inmunosupresión (Tabla 2).

Volumen de pacientes con TBC atendidos. En países desarrollados, el riesgo estimado de infección por $\mathrm{TBC}$ es menor al $0,2 \%$ para aquel PS que trabaja en hospitales con menos de 10 ingresos anuales por TBC o un caso cada 100 trabajadores de la salud. Este

PS: personal de salud 
riesgo aumenta progresivamente a medida que más pacientes son atendidos o más PS atiende estos pacientes, hasta llegar a riesgos de conversión (infección) de 1 a $10 \%$ en casos de más de 200 admisiones anuales por TBC o menos de 10 trabajadores por caso ingresado ${ }^{5}$. Las diferencias en las tasas anuales de infección o TBC activa entre países desarrollados y en vías de desarrollo, explica las mayores tasas de infección anual pesquisadas sistemáticamente en el PS en este último grupo de países. En estos casos, las tasas anuales de conversión tuberculínica (infección latente), sobrepasan habitualmente el 10\% del grupo bajo seguimiento y las tasas de incidencia de TBC activa se incrementan notoriamente, sobrepasando los 100 casos por 100.000 personas en riesgo ${ }^{6-8}$.

Ocupación del personal de salud. Varias funciones clínicas se asocian a un mayor riesgo de infección por TBC en el $\mathrm{PS}^{4}$. Los médicos y estudiantes de medicina tienen un mayor riesgo reportado de infección o enfermedad. Por ejemplo, en Perú, Bonifacio y cols, señalan una tasa anual de infección latente para internos y médicos en contacto con pacientes con TBC de $17 \%$, superior al valor reportado para la población general y $2 \%$ de casos anuales con enfermedad ${ }^{6}$. Esta situación también ha sido descrita en estudiantes de pre-grado de medicina en Brasil. En Río de Janeiro, hacia el año 1999, en un hospital con 270 ingresos anuales por TBC se pudo establecer un riesgo anual de infección de $3,9 \%$, siendo este riesgo, en un análisis multivariado, casi cuatro veces mayor en el grupo de estudiantes en contacto con pacientes versus el grupo sin contacto con ellos $(5,8 \text { versus } 1,6 \%)^{9}$. De la misma manera, la incidencia de casos de TBC activa entre los médicos residentes en un hospital general de México alcanzó un valor de 1.846 casos por 100.000 personas, incluso superior al resto de los médicos de la misma institución (860 por 100.000) ${ }^{8}$.

El grupo de enfermeras también presenta un mayor riesgo de infección o $\mathrm{TBC}$ activa respecto a otras funciones o profesiones entre el PS, en algunos casos superando al grupo médico ${ }^{4,10}$.

El personal paramédico y aquellos que participan en procedimientos de terapia respiratoria, también están expuestos a un mayor riesgo. En un estudio transversal con cerca de 10.000 pacientes con TBC, entre 16 y 64 años, en E.U.A., y analizando las profesiones u ocupaciones de los afectados, se pudo demostrar que el riesgo de TBC es más alto entre los ayudantes de enfermería y los terapeutas respiratorios que aplican inhalaciones. Mas aún, fue posible demostrar que el nivel socio-económico está asociado con este riesgo, observándose una mayor frecuencia de casos entre aquel PS de menor ingreso ${ }^{11}$. En un estudio cubano con trabajadores de un hospital psiquiátrico de La
Habana, el grupo con mayor riesgo de adquirir una TBC clínica resultó ser el de auxiliares generales, con un riesgo 8,21 veces mayor que el de la población general y 2,54 veces superior al grupo de enfermeras ${ }^{12}$. Este mismo perfil ha sido observado en otros trabajos, por ejemplo en Sudáfrica, con tasas sobre 1.100 casos de TBC activa por 100.000 personas para este grupo $^{13}$.

Lugar de trabajo del personal de salud. El sitio o lugar de trabajo es un importante determinante en el riesgo de infección o enfermedad por TBC. Actualmente, los pacientes con TBC son manejados en forma ambulatoria o en hospitales no dedicados específicamente a esta patología, y por lo tanto, el riesgo es transversal. Este riesgo involucra al personal de unidades respiratorias, al de los laboratorios de microbiología y al personal de anatomía patológica ${ }^{4}$.

En aquellas regiones donde aún se deriva a los pacientes a instituciones dedicadas en exclusividad al tratamiento de la TBC, es posible apreciar un riesgo incrementado para los funcionarios de esos hospitales. Por ejemplo, estudios llevados a cabo en la Federación Rusa han demostrado que el riesgo de TBC como enfermedad es más elevado en el personal que labora en hospitales para TBC que en consultorios o clínicas dedicados a ellos y a su vez más elevado que el resto del PS (1.216 casos por 100.000 personas-años para funcionarios de hospitales versus 317 por 100.000 personas-años para funcionarios de centros ambulatorios de TBC, en comparación con una tasa de 68 por 100.000 personas años para el resto del PS) ${ }^{14}$

La TBC es un hallazgo incidental en autopsias y expone al PS a adquirir una infección latente o enfermedad evidente ${ }^{15-17}$. El riesgo descrito es 100 a 200 veces más elevado que en la población general. Este mayor riesgo está asociado a la generación de aerosoles durante la autopsia (uso de sierra, disecciones al fresco), a la ausencia de sistemas de ventilación y la omisión del uso de barreras protectoras en el PS. En estas circunstancias, el riesgo es aún mayor que el observado para el PS en las salas de hospitalización antes del deceso del paciente. La posibilidad de contagiarse una TBC involucra también al personal forense, habiéndose descrito en ellos formas cutáneas por inoculación directa $^{15-17}$.

El riesgo de TBC también es mayor en el PS de los laboratorios de microbiología, especialmente entre aquellos que procesan las muestras respiratorias sin gabinetes de bio-seguridad y también ha sido documentado para funcionarios de laboratorios centrales ${ }^{18}$.

Oportunidad diagnóstica. El retraso en el diagnóstico de los pacientes expone a numerosas personas al riesgo de infección ${ }^{19}$. En diferentes reportes sobre brotes o TBC ocupacional, esta demora en el diagnóstico se ha extendido entre una y tres semanas desde el 
ingreso $^{19,20}$. La omisión de la sospecha se acrecienta a medida que la tasa de TBC decae en un país o que los ingresos de pacientes tuberculosos disminuyen en un hospital y aumenta la letalidad de la enfermedad porque retrasa el diagnóstico ${ }^{20}$. En ocasiones, la TBC se presenta en pacientes con otra enfermedad respiratoria o en pacientes inmunocomprometidos, los que son sometidos a procedimientos diagnósticos o terapéuti$\cos$ o son hospitalizados junto a otros pacientes, aumentando el riesgo de exposición hacia numerosos trabajadores. Además, este riesgo inadvertido también ocurre en la sala de autopsias. Las tasas de infección pueden superar el 10\% entre aquellos expues$\operatorname{tos}^{16}$. El retraso en la oportunidad diagnóstica también afecta al propio PS, con retardos mayores a lo observado en la población general ${ }^{12}$

Resistencia a fármacos anti-tuberculosos. La existencia de cepas multi-resistentes en un paciente mantiene su condición bacilífera a pesar de un tratamiento observado y favorece la aparición de brotes nosocomiales por cepas multi-resistentes ${ }^{21}$. En Chile, el porcentaje de multi-resistencia primaria (cepas resistentes a isoniazida y rifampicina en pacientes no tratados previamente) es muy baja y no supera el $0,5 \%{ }^{22}$

Sistemas de ventilación ambiental. Las malas condiciones de ventilación facilitan la transmisión de la TBC y ello explica su declinación con la mejoría en las condiciones de vivienda de la población. Los asilos, cárceles, sanatorios y hospitales antiguos, facilitan la concentración de numerosos pacientes y PS en luga-

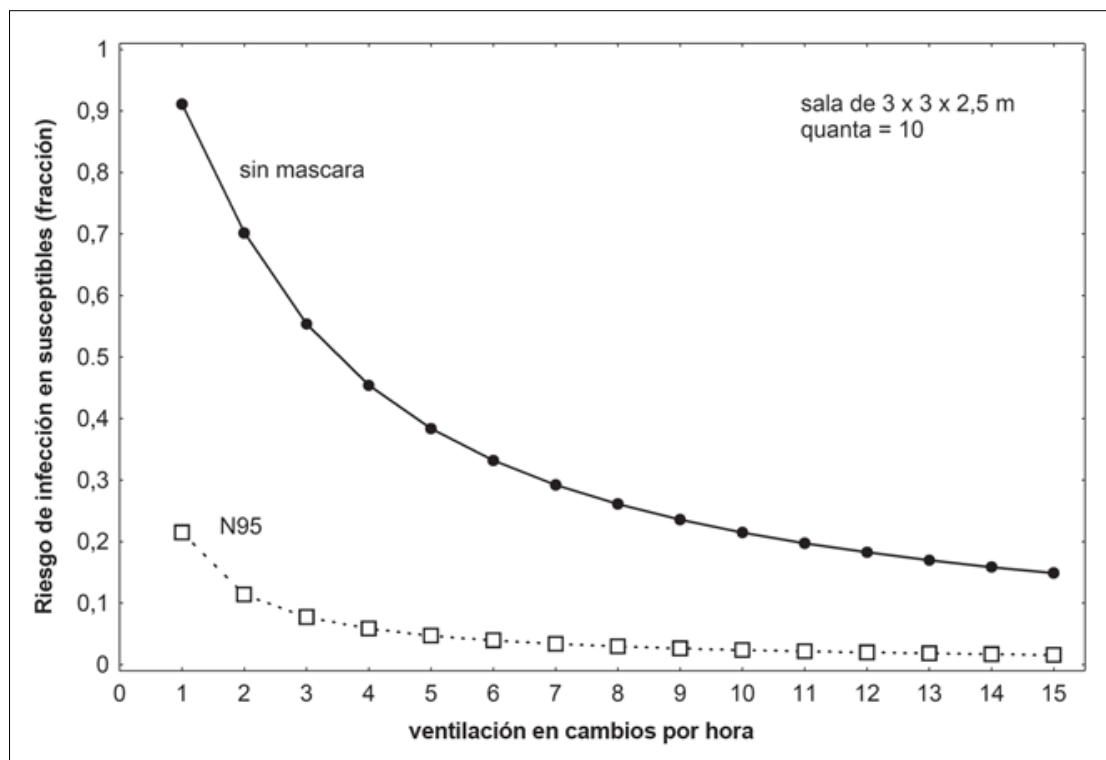

Figura 1. Riesgo teórico de $T B C$ en personas susceptibles en un jornada de ocho horas según variaciones en la ventilación de una pieza de 3 por 3 por 2,5 m, con o sin uso de mascarilla N95. La dosis infectante (quanta) se ha mantenido constante en 10. res cerrados. En la Figura 1 se puede apreciar cómo la ventilación de un espacio cerrado disminuye progresivamente el riesgo teórico de infección, aplicando la fórmula de Riley-Wells ${ }^{23}$. Por estas razones, en países industrializados se recomienda que los hospitales cuenten con salas generales con dos recambios por hora, seis en salas de aislamiento y 15 para salas de broncoscopia $^{24}$.

La evidencia más sustantiva sobre la importancia de los sistemas de ventilación en la adquisición de TBC nosocomial proviene un trabajo clásico desarrollado en Canadá entre diferentes hospitales, donde Menzies y cols, lograron demostrar, en un análisis multivariado, con un diseño prospectivo, que el riesgo de conversión tuberculínica en el PS de enfermería y kinesiólogos era mayor cuando ellos trabajaban en salas generales que tenían un menor recambio de aire por hora, respecto a aquellos que no convirtieron por trabajar en un lugar con mayor recambio de aire $(2,2$ versus 4,2 recambios por hora). El personal de kinesiología destinado a la atención en salas de broncoscopia también tuvo un mayor riesgo si se desempeñaba en un lugar con un menor número de recambios por hora respecto a los que trabajaban en sitios con un mayor número de recambio de aire por hora (5 versus 21$)^{24}$. El riesgo relativo de infectarse de $\mathrm{TBC}$ fue 3,4 veces superior para el PS que trabajaba en salas generales que tenían menos de dos recambios por hora respecto a aquellos que trabajaban en salas con dos o más recambios en un hora.

Aislamiento por aerosoles, barreras protectoras para el personal y protección en el laboratorio. La importancia de las precauciones (o aislamientos) por aerosoles para reducir el riesgo de contagio nosocomial ha sido evaluada en forma indirecta, mediante comparaciones antes-después o comparando las tasas de infección en el PS que labora en hospitales con diferente programas de protección. En este caso, el ítem aislamiento por aerosoles se ha aplicado en conjunto con otras medidas transversales de protección, tales como las máscaras de protección tipo N95 y las medidas de seguridad en el laboratorio de microbiología y por ello, no se puede estimar el impacto de ellas por separado.

En un estudio efectuado en Brasil, comparando las tasas de infección entre cuatro hospitales con diferencias en la intensidad de sus medidas de protección, se logró establecer que las tasas eran al menos el doble más altas en el PS que laboraba en hospitales con programas mínimos de protección respecto de los otros dos hospitales que aplicaban un programa más amplio (16 casos versus 7,8 por 1.000 personas-mes) ${ }^{10}$. Los hospitales con menor conversión presentaban como 
características el contar con una organización para la sospecha y tratamiento precoz, el uso de aislamiento en piezas individuales para los pacientes baciliferos, uso de campanas de bio-seguridad tipo II en el laboratorio de microbiología y de acceso restringido y la atención de pacientes con TBC en aislamientos con mascarillas tipo N95. Los hospitales con mayores tasas de conversión no tenían defensas algunas.

PS con inmunosupresión. El PS con inmunocompromiso tiene mayor riesgo de presentar enfermedad clínica y un desenlace fatal por esta enfermedad. Este fenómeno ha sido especialmente descrito para el PS afectado por infección con $\mathrm{VIH}^{25,26}$. En ciertos escenarios, hasta un tercio de los casos de TBC activa en el PS aparecen asociados a personal con infección por $\mathrm{VIH}^{25,27}$.

Desnutrición en el PS. En un estudio caso control desarrollado en la India, el PS con un índice de masa corporal $<19 \mathrm{~kg} / \mathrm{m}^{2}$ se asoció a un mayor riesgo de desarrollar TBC clínica ${ }^{28}$.

Condiciones del paciente que aumentan el riesgo. Diferentes condiciones del paciente aumentan el riesgo de contagio hacia el PS $^{29}$ (Tabla 3 ).

\section{Tabla 3. Condiciones del paciente con TBC activa} que aumentan el riesgo de contagio a PS

Presencia de tos
Lesiones cavitadas pulmonares
Enfermedad pulmonar con baciloscopia positiva
Compromiso laríngeo
Incapacidad para cubrirse la boca y nariz durante la tos
Aplicación incorrecta del tratamiento o abandono
Aplicación de procedimientos que generan aerosoles
(expectoración inducida, fibrobroncoscopia, nebulizaciones)
PS: personal de salud

¿Cuál es el riesgo descrito en Chile?. La alta cobertura de la vacuna BCG en Chile ha impedido hacer estudios de conversión tuberculínica y sólo se dispone de un estudio de TBC en el PS, publicado hace una década $^{30}$. En este estudio, efectuado el año 1995 con el PS de los hospitales San José, Sanatorio San José de Maipo, Instituto Nacional del Tórax y del Laboratorio de Micobacterias del Instituto de Salud Pública, se pudo demostrar una tasa de TBC aproximadamente seis veces superior a la de la población general del mismo grupo etario. A pesar de que este estudio tiene un sesgo de inclusión por el tipo de instituciones participantes, establece claramente la presencia de este riesgo en Chile. La mayor parte de los afectados fueron técnicos paramédicos y la mayor parte se desempeñaba en laboratorios, servicios de medicina o consultorios adosados. En un estudio reciente desarrollado por nuestro grupo entre el PS del Servicio de Salud Metropolitano Sur, se detectó un riesgo tres veces superior al de la población general (datos enviados a publicación).

El aporte de los estudios moleculares en la transmisión nosocomial de TBC. Diferentes interrogantes epidemiológicas han podido ser contestadas con el aporte de los estudios moleculares. En primer lugar, se ha podido establecer que no todos los casos de TBC activa en el personal de salud son adquiridos en el nosocomio sino que, como era de esperar, una fracción de ellos ocurre por reactivación de una infección latente antigua. En un trabajo reciente y utilizando métodos epidemiológicos y de tipificación molecular con la secuencia de inserción IS6110, comparando los resultados con bases de datos, De Vries y cols, lograron precisar que sólo $42 \%$ de los casos observados durante cinco años en el PS de los Países Bajos fue por adquisición nosocomial, $28 \%$ por contagio en la comunidad y el $30 \%$ restante por adquisición remota antes de una migración ${ }^{31}$. En un estudio similar con el PS de San Francisco, los autores lograron demostrar que, al menos $32 \%$ de los casos, estaban relacionados a una adquisición nosocomial ${ }^{32}$. No hay estudios extensos de esta naturaleza desarrollados en países de menos recursos que permitan evaluar el riesgo atribuible con mayor precisión. No obstante, en un trabajo desarrollado en India, se pudo determinar que en un corto período de tiempo ( $<1$ año) algunos de los casos hospitalizados en una misma institución y que aparentemente no estaban relacionados, estaban molecularmente relacionados y que ello era explicado por coincidencias en los períodos de hospitalización, no sólo en la misma sala sino que también en salas contiguas ${ }^{33}$.

Formas clínicas de la TBC. La presentación clínica de la TBC activa no difiere de la de otros pacientes, predominando la forma pulmonar con tos, expectoración, hemoptisis, fiebre y pérdida de peso $^{12,13,34}$. En algunas publicaciones se describe el predominio de las formas extra-pulmonares, especialmente ganglio$\operatorname{nar}^{35}$

Diagnóstico. Dos problemas diagnósticos deben ser resueltos en el caso de la TBC ocupacional. En primer lugar, contar con un sistema que permita un diagnóstico rápido en los pacientes hospitalizados con sospecha de TBC y, en segundo lugar, una estrategia que permita diagnosticar infección latente en el PS. En este último caso, se debe contar con una prueba 
de aplicación periódica. Las herramientas clínicas y de laboratorio en ambos casos no son las mismas (Tabla 4).

Diagnóstico de TBC en el paciente. El aspecto fundamental es la sospecha precoz y ello depende de la capacitación del PS, especialmente si la enfermedad se hace cada vez más infrecuente. Los laboratorios también deben organizarse para optimizar y acortar los tiempos de análisis e informe de las baciloscopias (tinción de Ziehl Neelsen). Esta tinción se encuentra ampliamente disponible, es fácil de realizar, de bajo costo, pero requiere personal entrenado para su lectura. La latencia en la entrega del informe es de pocas horas, tomando la lectura de la lámina unos 15 minutos. La técnica de fluorescencia con auramina-rodamina es un procedimiento alternativo que tiene, en contraste, una mejor sensibilidad y una lectura de sólo tres minutos por lámina, aunque con una menor especificidad.

Diferentes estrategias se han incorporado al laboratorio para acortar los tiempos de diagnóstico respecto al cultivo tradicional en el medio Löwenstein Jensen, que demora cuatro semanas o más. Estas estrategias incluyen los medios líquidos de cultivo, el uso de nuevas técnicas microscópicas y técnicas molecula-

\begin{tabular}{|lll|}
\hline \multicolumn{2}{c}{ Tabla 4. Problemas diagnósticos en TBC ocupacional } \\
\hline Parámetro & TBC en el paciente & TBC en el PS \\
\hline Problema & Diagnóstico de enfermedad & Diagnóstico de infección \\
Síntomas & Presentes & Habitualmente ausentes \\
\hline Requisitos del estudio & Sospecha precoz, diagnóstico & Vigilancia activa de los expues- \\
& rápido y sensible & tos con una prueba no inva- \\
& & sora, económica, de aplicación \\
& & periódica sensible y específica
\end{tabular}

Tabla 5. Métodos de diagnóstico acelerado de Mycobacterium tuberculosis

\section{Método}

Cultivo acelerado

Nuevas técnicas microscópicas (MODS: microscopic observation of drug susceptibility)

Métodos moleculares

\section{Comentarios}

Medios líquidos en varios formatos disponibles, algunos no radioactivos, acortan el diagnóstico permitiendo identificación en 8 a 13 días de incubación. Lectura manual o automatizada

Utilizan microscopio de fase invertida para lectura de cultivos en medios líquidos con o sin fármacos anti-TBC asociados. Tiempo medio de positividad 7 días. Bajo costo y sensibilidad superior al cultivo en medios líquidos o sólidos

Varios métodos disponibles ya sea comerciales o "hechos en casa", basados en RPC. Los métodos "hechos en casa" pueden tener problemas de reproducibilidad. RPC tiene especificidad subóptima en muestras con baciloscopias negativas. Algunos formatos permiten estudio de susceptibilidad a fármacos anti-TBC $\operatorname{res}^{36}$ (Tabla 5). Entre ellas, la técnica MODS es la más reciente y promisoria.

Diagnóstico en el personal de salud. A diferencia del problema planteado con pacientes, en el caso de PS se requiere una estrategia que permita el diagnóstico de infección en ausencia de síntomas $\mathrm{y}$, en sus defectos, la búsqueda activa de PS con TBC clínica. Para ello se pueden utilizar parte o la totalidad de las siguientes estrategias (Tabla 6).

En los países en vías de desarrollado, el uso de la prueba de tuberculina es complicado por el amplio uso de la vacuna BCG. Un alto porcentaje del PS presenta una prueba positiva al inicio de su trabajo, debido al antecedente de vacunación. Esta cifra supera, habitualmente, el $50 \%$, dejando una fracción menor que puede ser seguida para evaluar tasas de conversión tuberculínica ${ }^{7}$. Este antecedente impide una estrategia basada en el empleo de la tuberculina.

La prueba de tuberculina no sólo está influenciada por el antecedente de vacuna $B C G$, sino que también por la variabilidad en la administración del preparado o en su lectura, por la exposición a micobacterias atípicas ambientales, hecho especialmente importante en zonas geográficas tropicales, también por el estado inmune del huésped (bajo uso de corticosteroides, desnutrición, infección por VIH u otras) y finalmente por el efecto booster o de refuerzo de la misma prueba. En este caso, si una segunda prueba se hace poco después de la primera, una fracción de las personas tendrá una prueba positiva por el reclutamiento de linfocitos de memoria entre las dos pruebas. Por ello, si se opta por un seguimiento basado en la prueba de tuberculina en aquellos inicialmente negativos, se debe repetir esta prueba, entre una y tres semanas después, para identificar la fracción del PS que hace una prueba positiva con este refuerzo y que representan falsos negativos de la prueba original. Esta secuencia se conoce bajo el concepto de doble test ${ }^{37,38}$. La prueba de tuberculina requiere de una aplicación y lectura estandarizada y debe ser efectuada por personal entrenado. El PS se considera en riesgo intermedio para adquirir TBC y la recomendación es usar una lectura $\geq 10 \mathrm{~mm}$ como positiva ${ }^{39}$. Cuando se efectúa un seguimiento, un incremento $\geq 6 \mathrm{~mm}$ entre las dos pruebas se considera evidencia de conversión.

\section{Tabla 6. Estrategias diagnósticas en el personal} de salud

Prueba de conversión de tuberculina

Pruebas de liberación de interferón gama

Tamizaje de sintomáticos respiratorios con radiografía de tórax y baciloscopias 
En años recientes se ha introducido una prueba basada en la liberación de gama-interferón desde linfocitos circulantes. Esta prueba conocida como

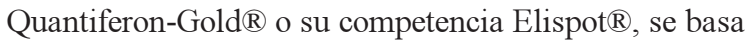
en que las células $\mathrm{T}$ de los individuos previamente sensibilizados con antígenos tuberculosos, producen esta citoquina cuando se re-encuentran con antígenos propios de Mycobacterium tuberculosis. Ofrece mejor especificidad que la prueba de tuberculina ya que no presenta una reacción cruzada con micobacterias atípicas o con $M$. bovis y por ello no es afectada por la vacuna $\mathrm{BCG}$, un derivado de $M$. bovis. Tiene la ventaja de requerir sólo una visita del paciente (al momento de la extracción de sangre), los resultados se encuentran disponibles en $24 \mathrm{~h}$ posterior a la toma de muestra y no está sometida al riesgo de errores humanos en la lectura e interpretación de los resultados. Los riesgos de esta prueba están dados por errores en la obtención y transporte de muestras al laboratorio, o en la corrida y lectura de los resultados, especialmente cuando ellos se encuentran cerca del punto de corte. Su mayor inconveniente es el alto precio ${ }^{40,41}$. Las pruebas seriadas con este examen aún se encuentran en delineación y no hay una definición respecto al incremento que debe ser considerado como indicador de infección recien$\mathrm{te}^{42}$.

La especificidad de esta prueba para detectar casos de infección latente entre alumnos de enfermería vacunados con BCG fue de $98,1 \%$ en un estudio efectuado en Japón ${ }^{43}$. No se dispone de datos de sensibilidad ya que no existe un estándar de oro para esta condición, pero tiene una mejor correlación que la tuberculina con la magnitud de la exposición ${ }^{44}$.

Tratamiento anti-tuberculoso. El abandono del tratamiento específico, la existencia de casos asociados a cepas multi-resistentes (MR) e incluso casos letales de TBC, no son fenómenos ajenos en la evolución y tratamiento del PS con TBC activa. En un estudio en Sudáfrica, la tasa de abandono alcanzó al 6,6\% y hubo $3 \%$ de casos con cepas $\mathrm{MR}^{13}$.

Medidas de prevención de transmisión de TBC nosocomial y su impacto. Las medidas de prevención recomendadas para evitar la infección en el PS están organizadas en tres niveles complementarios ${ }^{29}$ (Tabla 7). Algunas de estas medidas se revisan a continuación.

Sospecha precoz. El retraso en el diagnóstico de pacientes hospitalizados con TBC ha sido un factor importante en la transmisión hacia el PS y en la generación de brotes ${ }^{5,19,20}$. El PS debe recibir educación sobre la sospecha de este diagnóstico y aplicar sistemáticamente el estudio bacteriológico a todo paciente que se hospitaliza y que tiene síntomas respiratorios por más de dos semanas, en forma independiente a la causa de ingreso.

Ventilación y mascarillas tipo N95. En la Figura 2, se presentan diferentes dosis de unidades infectantes y las variaciones en el riesgo, de acuerdo a la ventilación y uso de mascarilla N95. En este caso se puede observar que a bajas dosis infectantes (por ejemplo a pocos días de iniciado un tratamiento anti TBC), el

Tabla 7. Medidas para prevenir la transmisión de TBC nosocomial

$\begin{array}{ll}\text { Medidas de prevención } & \text { Componentes } \\ \text { Administrativas } & \text { Diseñar un plan de trabajo local con evaluación del riesgo } \\ & \text { Sospecha y diagnóstico precoz en pacientes } \\ & \text { Aislamiento en personas con alta sospecha o con diag- } \\ & \text { nóstico de TBC pulmonar } \\ & \text { Desinfección de equipos de riesgo (fibro-broncoscopio, } \\ & \text { laringoscopio) } \\ & \text { Educación y entrenamiento del PS } \\ & \text { Tamizaje para diagnóstico de infección y enfermedad en } \\ & \text { el PS } \\ & \text { Manejo de muestras respiratorias en campanas de bio- } \\ & \text { seguridad cuando se efectúan cultivos } \\ & \text { Sistemas de ventilación o depuración del aire en aquellos } \\ \text { Infraestructura } & \text { lugares con pacientes bacilíferos pulmonares } \\ \text { Protección personal } & \text { Mascarillas tipo N95 y entrenamiento del PS } \\ & \text { Aplicar etiqueta de tos a los pacientes } \\ & \text { Evitar que el PS con infección por VIH participe en la } \\ \text { PS: pención de pacientes con TBC activa }\end{array}$

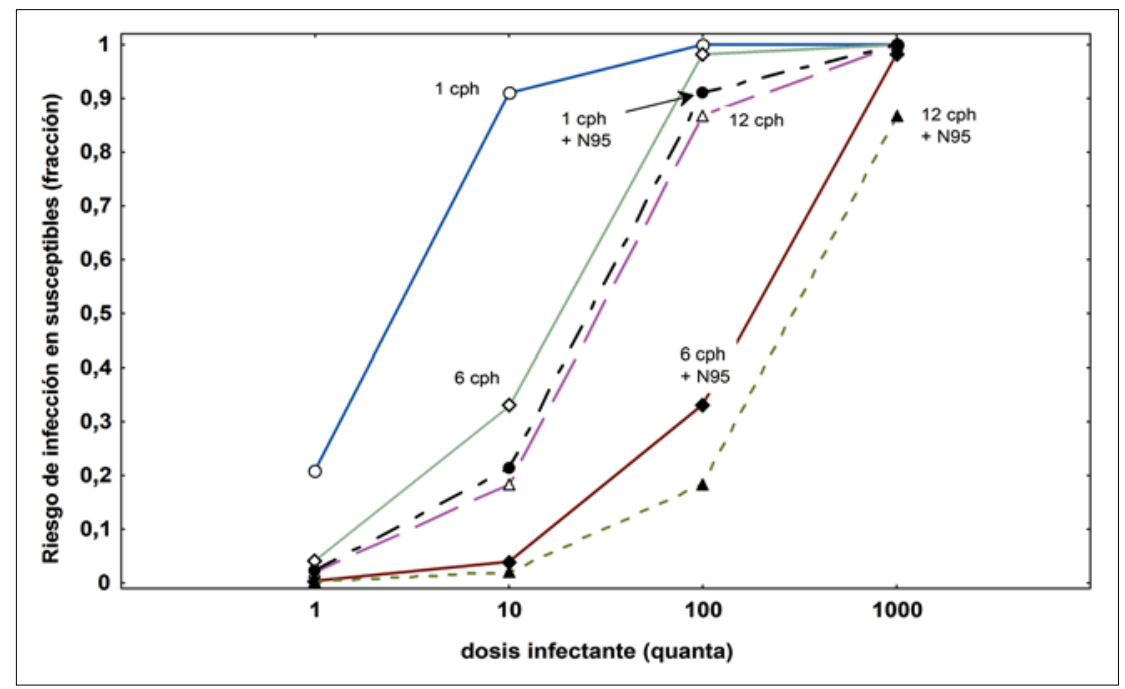

Figura 2. Distribución teórica del riesgo de TBC en una jornada de ocho horas según diferentes dosis infectantes, cambios en la ventilación (cph: cambios por hora) y uso de mascarilla N95 en una sala de 3 por 3 por 2,5 m conteniendo un paciente con TBC bacilífera. 
efecto protector de la mascarilla no es importante. En contraste, en situaciones de altas concentraciones de aerosoles con M. tuberculosis (por ejemplo en autopsias), la ventilación y las mascarillas tienen límites para otorgar una protección al PS ${ }^{45}$.

La dosis infectante o quanta asociada a un paciente con TBC pulmonar cavitada (sin tratamiento) es de 13 , de 250 para procedimientos endoscópicos pulmonares y sobre 1.000 para autopsias de pacientes con TBC.

Las mascarillas tipo N95 permiten que el PS tenga una menor exposición a aerosoles infectantes cuando atiende pacientes con TBC pulmonar bacilífera. Permite la filtración y retención eficiente de partículas menores a $5 \mu$. Debe ser utilizada apropiadamente, sin filtraciones de aire por los costados y el PS debe ser entrenado en su uso. Es desechable y más costosa que las mascarillas quirúrgicas comunes; estas últimas no otorgan protección alguna contra los aerosoles que contienen este agente. Las mascarillas quirúrgicas sólo deben se utilizadas en el paciente cuando abandona la sala para procedimientos o exámenes para capturar secreciones mayores y disminuir la posibilidad de generar aerosoles

Tamizaje para el diagnóstico de infección y de enfermedad en el PS. En países con una alta cobertura de vacuna $\mathrm{BCG}$ es impracticable la realización de un seguimiento anual con una prueba de tuberculina para detectar conversión e indicar una profilaxis con isoniazida. Ello se debe a que sobre $50 \%$ de la población tiene una prueba inicial positiva, impidiendo un trabajo de seguimiento. En estas condiciones, es más importante la detección precoz de enfermedad clínica, capacitando al PS para que se realice un estudio si tiene tos persistente por más de dos a tres semanas. El equipo de salud debe efectuar una evaluación clínica, radiológica y microbiológica para descartar una TBC activa. En países desarrollados se puede plantear un algoritmo basado en la prueba de tuberculina o su equivalente moderno, una prueba de liberación de interferón gama en linfocitos del paciente estimulados por antígenos específicos de M. tuberculosis.

Evitar el riesgo de exposición en el personal de salud portador de infección por VIH. La aparición de casos de TBC en el PS infectado con VIH, algunos de ellos por cepas multi-resistentes y letales en E.U.A., advirtió del serio riesgo de exposición que tenían estas personas como trabajadores de la salud y determinó el desarrollo de políticas para evitar su participación en la atención de pacientes o áreas de riesgo. La situación es dramática en las regiones más pobres, especialmente en África, debido a la alta prevalencia de la infección por VIH. La sugerencia oficial internacional es el tamizaje del PS para VIH en estas áreas o funciones. Sin embargo, esta estrategia se contrapone con el derecho de las personas a decidir su consentimiento para este examen. En África, este tamizaje se asoció a abandono familiar o incluso, actos de violencia intrafamiliar, en el caso de mujeres con una prueba seropositiva; por ello, algunos expertos recomiendan reemplazar el tamizaje obligatorio por una invitación a la realización de esta prueba $^{46}$.

Normas de bio-seguridad en laboratorios de microbiología que procesan cultivos. La generación de aerosoles durante la preparación de cultivos de micobacterias expone al PS al riesgo de TBC y por ello, se recomienda que este proceso se efectúe en campanas de bio-seguridad. No obstante, si en el lugar de trabajo sólo se realizan tinciones, no es necesario aplicar estas campanas. En estos casos, deben seguirse las siguientes instrucciones: abrir el recipiente despacio; poner el mechero encendido entre el funcionario (trabajando con brazo extendido) y la placa durante la preparación del frotis; esperar que el frotis se seque en forma espontánea, sin flamearlo (genera menos aerosoles).

Impacto. Las diferentes medidas de control y prevención de transmisión nosocomial de $M$. tuberculosis han sido evaluadas en varios escenarios mundiales, tanto en países desarrollados como en aquellos en desarrollo. El esquema de evaluación habitual ha sido la medición de tasas de conversión o de TBC activa antes-después en el PS o la comparación de estas mismas variables entre hospitales que han implementado medidas, respecto a aquellos que lo han hecho en forma parcial o no las han implementado.

Por ejemplo, Yanai y cols, en un hospital regional de Tailandia, lograron disminuir la tasa de conversión anual de tuberculina desde 9,3 casos por 100 personas-año, en los año 1995-1997 a 6,4 el año 1998 y luego a 2,2 casos por 100 personas-año en el año 1999, gracias a la implementación de un programa global que consideró capacitación y organización del PS, sospecha y diagnóstico precoz, aislamiento por aerosoles para los pacientes (incluyendo dos salas individuales con presión negativa, máscaras tipo N95 para el personal y etiqueta de tos para los afectados) y además condiciones de bio-seguridad en el laboratorio de microbiología ${ }^{7}$ (Tabla 8)

También se reportó una declinación en la tasa anual de conversión tuberculínica en el PS en Turín, Italia, luego de implementar en toda la región un programa de prevención de TBC ocupacional. Los valores descendieron desde 2,2 casos de conversión a 0,8 eventos por 100 personas-año ${ }^{47}$. Las medidas de prevención fueron semejantes a las sugeridas por el CDC. El impacto fue global entre los diferentes profesionales y ocupaciones del equipo de salud y no estuvo asociado a una declinación de TBC en la zona ya que esta variable no cambió (Tabla 8). 
Tabla 8. Impacto de diferentes programas de intervención para disminuir el riesgo de infección latente o TBC ocupacional

\begin{tabular}{|c|c|c|c|c|c|}
\hline País y autor & Período & Lugar & Intervención & $\begin{array}{l}\text { Resultado en ta: } \\
\text { Inicio }\end{array}$ & $\begin{array}{c}\text { is de conversión } \\
\text { Final }\end{array}$ \\
\hline $\begin{array}{l}\text { Tailandia } \\
\text { Yanai }\end{array}$ & 1995 a 1999 & Hospital local & Tipo CDC & $\begin{array}{l}9,3 \% \text { anual } \\
1995 \text { a } 1997\end{array}$ & $\begin{array}{c}2,2 \% \text { anual } \\
1999\end{array}$ \\
\hline $\begin{array}{l}\text { Malawi } \\
\text { Harries }\end{array}$ & 1996 у 1999 & Varios hospitales & Normas locales & $\begin{array}{c}3,7 \% \text { anual de TBC } \\
1996^{*}\end{array}$ & $\begin{array}{c}3,2 \% \text { TBC } \\
1999^{*}\end{array}$ \\
\hline $\begin{array}{l}\text { Italia } \\
\text { Baussano }\end{array}$ & 1997 al 2004 & Hospitales ciudad de Turín & Tipo $C D C$ & $\begin{array}{l}2,2 \text { por } 100 \mathrm{p}-\mathrm{a} \\
\text { Antes del } 2000\end{array}$ & $\begin{array}{l}\text { 0,8 por } 100 \text { p-a } \\
\text { Después del } 2000\end{array}$ \\
\hline $\begin{array}{l}\text { E.U.A. } \\
\text { Bangsberg }\end{array}$ & 1992 a 1994 & Hospital en New York & $\begin{array}{c}\text { Tipo CDC (sin detalles } \\
\text { sobre Laboratorio } \\
\text { de Microbiología) }\end{array}$ & $\begin{array}{c}5,8 \text { por } 100 \mathrm{p}-\mathrm{a} \\
1992\end{array}$ & $\begin{array}{c}0,0 \text { por } 100 \mathrm{p}-\mathrm{a} \\
1994\end{array}$ \\
\hline $\begin{array}{l}\text { E.U.A. } \\
\text { Blumberg }\end{array}$ & 1992 a 1997 & Hospital de Atlanta & Tipo CDC & $\begin{array}{c}5,98 \text { por } 100 \text { p-a } \\
1992\end{array}$ & $\begin{array}{c}1,0 \text { por } 100 \text { p-a } \\
1993 \text { a } 1997\end{array}$ \\
\hline $\begin{array}{l}\text { E.U.A. } \\
\text { Wenger }\end{array}$ & 1990 a 1992 & $\begin{array}{c}\text { Miami } \\
\text { Sala VIH, Brote TBC-MR }\end{array}$ & Tipo CDC & $\begin{array}{c}28 \% \text { conversión en PS } \\
\text { sala } \mathrm{VIH}\end{array}$ & $\begin{array}{c}\text { 0\% conversión en PS } \\
\text { sala } \mathrm{VIH}\end{array}$ \\
\hline $\begin{array}{l}\text { E.U.A. } \\
\text { Maloney }\end{array}$ & 1991 & $\begin{array}{c}\text { New York } \\
\text { Brotes TBC-MR }\end{array}$ & Tipo CDC & $\begin{array}{l}17 \% \text { PS en contacto } \\
\text { con pacientes con TBC }\end{array}$ & $\begin{array}{c}5 \% \text { PS en contacto } \\
\text { con pacientes con TBC }\end{array}$ \\
\hline
\end{tabular}

En E.U.A., Bangsberg y cols, reportaron una declinación importante y sostenida en el PS de un hospital de New York, luego de haber introducido varias medidas sugeridas por el $\mathrm{CDC}^{48}$. De la misma manera, Blumberg y cols, también reportaron un éxito transversal en la disminución de las tasas de conversión entre médicos con la implementación de las medidas del $\mathrm{CDC}^{49}$ (Tabla 8).

No obstante estos éxitos en la disminución del riesgo de conversión tuberculínica, estos programas no han logrado demostrar una reducción significativa de los casos de TBC activa entre el PS4.

Los programas de prevención también han tenido impacto para reducir y controlar brotes de infección nosocomial por cepas multi-resistentes en pacientes inmunocomprometidos o inmunocompetentes. En un estudio efectuado en Miami, el programa de intervención permitió reducir la tasa de TBC por cepas multiresistentes (TBC MR) en pacientes hospitalizados con infección por VIH y también disminuir la tasa de conversión tuberculínica en el $\mathrm{PS}^{50}$ (Tabla 8). En otra experiencia, se redujo el riesgo de TBC MR nosocomial en la ciudad de New York y, al mismo tiempo, disminuyó el riesgo de conversión tuberculínica en el PS asignado a atención de pacientes con $\mathrm{TBC}^{21}$ (Tabla 8).

En un estudio desarrollado en Malawi, con normas locales en un país con limitaciones de recursos, no se pudo demostrar una reducción significativa en la tasa anual de TBC en el PS. Estas medidas optimizaban los tiempos de diagnóstico pero no incluyeron, por razones económicas, aislamientos individuales o uso de mascarillas N95 ${ }^{51}$ (Tabla 8). Además, un bajo porcentaje de los hospitales tuvo adherencia completa a las normas.

Todos estos antecedentes indican que las intervenciones sugeridas por el CDC son exitosas para reducir el riesgo de TBC nosocomial y ocupacional, incluyendo casos con TBC MR. No obstante, los países en vías de desarrollo tienen serias dificultades para implementar estas estrategias de protección, debido a las dificultades económicas para asegurar un diagnóstico o sospecha precoz, para asegurar los medicamentos, la asignación de insumos de protección o para hacer modificaciones estructurales ${ }^{18,28}$

La ventilación natural es la forma predominante de manejo del aire en los hospitales de países en vías de desarrollo. No obstante, esta forma de ventilación permite recambios de aire con la consiguiente disminución del riesgo. Por ejemplo, en un estudio reciente, Escombe y cols evaluaron, en Perú, la ventilación natural en las salas de ocho hospitales de Lima, mediante la técnica de la depuración de $\mathrm{CO}_{2}$ en el aire ${ }^{52}$. Las salas demostraron tener un recambio de aire por hora (mediana) cuando las puertas y ventanas estaban cerradas, 7,6 recambios con una ventana o puerta abierta y 20 recambios cuando la ventana y la puerta estaban simultáneamente abiertas. Además, los edificios más antiguos tenían techos más altos y poseían mayores niveles de ventilación espontánea cuando fueron comparados con edificios más nuevos con techos más bajos (40 versus 17 recambios por hora con puertas y ventanas abiertas). El riesgo de contagio estimado fue 
de $97 \%$ en las personas susceptibles en una sala llena de pacientes con TBC bacilífera antes del tratamiento y sin ventilación, de $33 \%$ en salas de hospitales nuevas y de $11 \%$ en las salas de hospitales más viejos. El riesgo de contagio teórico en una sala de presión negativa con 12 recambios por hora resultó ser de $39 \%$, valor superior a las salas naturalmente ventiladas. Estos datos señalan que las edificaciones antiguas ofrecen cierto nivel de protección cuando se acostumbra a ventilar las salas que albergan pacientes con TBC. De esta manera, la existencia de una sala para pacientes con TBC no contraindica el hecho de que sea ventilada, sino solamente que no debe ser ventilada al pasillo del hospital. Estos mismos autores señalan que, a pesar de contar con salas de aislamiento con presión negativa, ellas no funcionaban adecuadamente debido a la falta de un programa de mantención ${ }^{52}$.

Vacuna BCG en el personal de salud. El diseño de una estrategia para prevenir la transmisión de TBC nosocomial fue realizado desde países desarrollados donde no se aplica la vacuna BCG. En contraste, la mayor parte de los países en vías de desarrollo aplica en la infancia rutinariamente la vacunación anti-tuberculosis.

La vacuna BCG tiene una eficacia protectora aproximada de $50 \%$ para TBC activa, de $64 \%$ para casos de meningitis y de $71 \%$ para prevenir muertes por esta causa $^{53}$. Es difícil realizar ensayos randomizados de eficacia protectora en el PS, debido a que el mayor riesgo se concentra en países donde ya se aplica la vacuna. En un estudio aplicando un modelo de decisión, se concluyó que la vacuna BCG es más efectiva que el seguimiento anual con la prueba de tuberculina para prevenir casos de TBC activa en el PS y muertes por esta causa. Para una cohorte de 100.000 personas seguidas a 10 años, el contar con la vacuna permite reducir aproximadamente en 49\% los casos de TBC y en $44 \%$ las muertes por esta condición respecto a no hacer ninguna intervención. En contraste, la prueba anual con tuberculina seguida de profilaxis con isoniazida en los casos con conversión permite sólo reducir en $10 \%$ los casos clínicos y no tiene impacto alguno en reducir las muertes por esta condición ${ }^{53}$. En este análisis se debe recordar que el riesgo de muerte es bajo para los casos tratados de TBC, no superior al $4 \%$ (en ausencia de cepas multi-resistentes o pacientes inmunocomprometidos). La vacuna protege además contra la infección o enfermedad por cepas resistentes. No obstante estos alentadores resultados, otro estudio similar arrojó resultados discrepantes, favoreciendo el uso de una estrategia basada en el seguimiento con la prueba de tuberculina ${ }^{54}$

Estos datos indican que el PS de aquellos países donde se aplica esta vacuna con altos grados de co- bertura, está de alguna manera protegido antes de iniciar sus labores. La OMS no recomienda la revacunación ya que no aporta mayor eficacia protectora ${ }^{46}$.

\section{Regulaciones y situación en Chile}

Luego de la creación del Programa Nacional de Control de la TBC (PCT) en 1973, se ha observado un proceso de sostenida disminución de los casos, con una tasa de reducción de $3,6 \%$ entre los años 2000 y 2005, y una incidencia de morbilidad de 15,7/100.000 habitantes durante ese último año ${ }^{55}$. Esto ha contribuido a que el número de pacientes hospitalizados sea cada vez menor, a que sean excepcionales los casos de TBC multi-resistente y a que sea un problema sólo ocasional en la población infantil.

En nuestro país existen dos documentos normativos respecto a bio-seguridad en $\mathrm{TBC}$, el primero se encuentra en el capítulo XIII del PCT y el otro ha sido desarrollado por el Programa de Infecciones Intrahospitalarias (PIIH) del Ministerio de Salud ${ }^{56}$. Las medidas administrativas propuestas por el PCT están ampliamente respaldadas en la literatura médica y recomendaciones internacionales. Se basan en evitar la presencia inadvertida de un caso de $\mathrm{TBC}$, fomentando la sospecha clínica y diagnóstico precoz para evitar la transmisión. Sin embargo, en los aspectos de manejo ambiental y medidas de protección personal existen algunas discordancias entre ambas normativas y ninguna se pronuncia sobre la aplicabilidad de ellas en la atención pediátrica (Tabla 9)

Los aspectos discordantes más llamativos se refieren a que

- El PIIH exige salas de presión negativa mientras el documento del PCT admite la posibilidad de hospitalización incluso en sala compartida;

- Adaptaciones de infraestructura para salas de fibrobroncoscopia y procedimientos respiratorios;

- La exigencia o no exigencia del tamizaje de infección por VIH en el PS que trabajará en lugares de riesgo;

- El ámbito de aplicación de mascarilla N95 y el seguimiento del PS.

Por otra parte, el PCT contempla una dosis de refuerzo de la vacuna $\mathrm{BCG}$ al PS con una prueba de tuberculina inicial negativa, una estrategia que la OMS ha descartado ya que no se ha observado beneficio adicional con nuevas inmunizaciones ${ }^{46,57}$. De esta manera, la búsqueda de PS con una prueba de tuberculina negativa en Chile, al parecer no está dirigida a identificar a un grupo para seguimiento.

En ambas normativas existen aspectos no resueltos, tales como las recomendaciones nacionales post 
exposición accidental del PS a paciente bacilífero y la implementación de sistemas de supervisión y evaluación del cumplimiento de la normativa. Estos temas debieran ser abordados en actualizaciones de ambos documentos, así como los aspectos discordantes antes mencionados.

Otras regulaciones. En la mayoría de los países europeos las guías recientes recomiendan que, en los lugares de baja prevalencia, se enfatice la importancia de adecuadas medidas de bio-seguridad, incluyendo salas con presión negativa ${ }^{58}$. Sin embargo, en países como el Reino Unido se aplican medidas que difieren en algunos aspectos a aquellas recomendadas por el CDC. Los más importantes son el ámbito de aplicación de las salas con presión negativa y el uso de mascarillas N95, medidas que se reservan sólo para casos de TBC multi-resistente ${ }^{59}$. Es así como los pacientes con TBC causada por cepas susceptibles son hospitalizados en habitaciones individuales, sin que el PS utilice mascarilla N95 e incluso sin tipo alguno de mascarilla ${ }^{60}$ (Tabla 10).

Otros aspectos. El alta precoz desde el hospital de pacientes con TBC pulmonar bacilíferos ha sido resistido en países desarrollados por la existencia de casos con cepas multi-resistentes. Por ello, se espera que las baciloscopias estén negativas ya que ello permite inferir el control de la enfermedad. No obstante, en nuestro medio, la prevalencia de cepas multi-resistentes es baja y, por lo tanto, no existe impedimento para considerar un alta precoz, siempre y cuando el paciente quede contactado con un centro de atención primaria para su seguimiento.
Tabla 9. Elementos discordantes en las regulaciones nacionales sobre manejo de pacientes con TBC

\begin{tabular}{|c|c|c|}
\hline Componente & Programa IIH & Programa TBC \\
\hline Laboratorio & $\begin{array}{l}\text { No especifica claramente } \\
\text { campana de bioseguridad }\end{array}$ & $\begin{array}{l}\text { Manejo apropiado de muestras } \\
\text { en Laboratorio, incluyendo ga- } \\
\text { binete en caso de cultivos }\end{array}$ \\
\hline Infraestructura & $\begin{array}{l}\text { Sala con presión negativa } \\
\text { para paciente bacilífero } \\
\text { hospitalizado } \\
\text { No menciona características } \\
\text { sobre espacios de toma de } \\
\text { muestras, FBC o procedi- } \\
\text { mientos respiratorios }\end{array}$ & $\begin{array}{l}\text { Obtención de muestras en salas } \\
\text { ventiladas } \\
\text { Hospitalización de pacientes en } \\
\text { salas bien ventiladas, incluso sala } \\
\text { común } \\
\text { Propone sistemas de ventilación } \\
\text { para salas de fibro-broncoscopia } \\
\text { o procedimientos relacionados } \\
\text { a vía aérea }\end{array}$ \\
\hline $\begin{array}{l}\text { Tamizaje para VIH } \\
\text { y otras condiciones }\end{array}$ & $\begin{array}{l}\text { No contempla prueba para } \\
\text { infección por VIH en el PS }\end{array}$ & $\begin{array}{l}\text { Prueba para infección por VIH, } \\
\text { tuberculina y Rx de tórax obliga- } \\
\text { toria en personal o alumnos que } \\
\text { pueden trabajar en condiciones } \\
\text { de "riesgo" Señala exclusión del } \\
\text { PS inmunosuprimido en zonas } \\
\text { de riesgo }\end{array}$ \\
\hline Protección personal & No precisa tipo de mascarilla & $\begin{array}{l}\text { Uso N95 en autopsias y en salas } \\
\text { de procedimientos de vía aérea } \\
\text { No obliga a su uso en atención } \\
\text { de pacientes en salas } \\
\text { Considera incluso el uso de mas- } \\
\text { carillas quirúrgicas en el PS que } \\
\text { participa en fibro-broncoscopia }\end{array}$ \\
\hline Control anual & $\begin{array}{l}\text { Especificado para PS en riesgo, } \\
\text { no especifica como }\end{array}$ & No especificado \\
\hline Otros & & $\begin{array}{l}\text { Señala vacuna } B C G \text { en casos con } \\
\text { prueba negativa tuberculina }\end{array}$ \\
\hline
\end{tabular}

Tabla 10. Comparación entre las medidas de manejo ambiental y protección personal recomendadas por las diferentes guías vigentes

\begin{tabular}{|c|c|c|c|c|}
\hline Medida & Programa IIH & Programa TBC & Guía CDC & Reino Unido \\
\hline Habitación individual & Siempre & Permite sala compartida & $\begin{array}{l}\text { Siempre } \\
\text { Recomienda planificar } \\
\text { número de habitaciones } \\
\text { necesarias según ingresos } \\
\text { con TBC }\end{array}$ & Siempre \\
\hline Presión negativa & $\begin{array}{l}\text { Recomienda salas con } \\
\text { presión negativa }\end{array}$ & No se pronuncia & $\begin{array}{l}\text { Siempre } \\
\text { Recomienda programa } \\
\text { adecuado de mantención }\end{array}$ & Sólo en TBC multi-resistente \\
\hline Uso de mascarilla & No precisa tipo de mascarilla & $\begin{array}{l}\text { Permite uso de mascarilla } \\
\text { quirúrgica, pero diferencia } \\
\text { riesgo }\end{array}$ & Mascarilla N95 & Sólo en TBC multiresistente \\
\hline $\begin{array}{l}\text { Salas de broncoscopia y } \\
\text { autopsias }\end{array}$ & No se pronuncia & $\begin{array}{l}\text { Permite uso de mascarilla } \\
\text { quirúrgica }\end{array}$ & Mascarilla N95 & $\begin{array}{l}\text { No precisado en el } \\
\text { documento }\end{array}$ \\
\hline
\end{tabular}




\section{Resumen}

La tuberculosis (TBC) es un riesgo ocupacional que explica entre 5 y 5.361 casos por 100.000 personas en el personal de salud (PS) por sobre la población general en países en vías de desarrollo. Cada caso clínico implica que han ocurrido muchos casos de infección. Esta infección puede ser detectada por la prueba de conversión de tuberculina entre personas no vacunadas o por prueba de liberación de gama interferón. Los factores de riesgo incluyen la frecuencia de pacientes con TBC atendidos, la función y lugar de trabajo del PS, retraso en la sospecha diagnóstica, atención de pacientes con cepas multi-resistentes, sis- temas de ventilación limitados, falta de aplicación de precauciones por aerosoles y PS con inmunosupresión o desnutrición. Los estudios moleculares han permitido aclarar que sólo 32 a $42 \%$ de los casos en el PS responde a adquisición ocupacional. Las medidas útiles para prevenir este riesgo incluyen un conjunto de disposiciones administrativas, de infraestructura y precauciones en el personal que han permitido reducir el riesgo de infección, y en ocasiones el de TBC clínica, en el PS. En Chile existen actualmente dos normas reguladoras sobre la materia, una del Programa Nacional de la TBC y el otro del Programa de Infecciones Intrahospitalarias, las que difieren en algunos aspectos sustantivos y que ameritan una revisión.

\section{Referencias}

1.- Valenzuela M T. Recomendaciones para la prevención y control de la tuberculosis en el personal de salud. Rev Chil Infect 1998; 15 : 234-41.

2.- Riley R L. Airborne infection. Am J Med 1974; 57: 466-75.

3.- Tam C M, Leung C C. Occupational tuberculosis: a review of the literature and the local situation. Hong Kong Med J 2006; 12: 448-55.

4.- Joshi R, Reingold A L, Menzies D, Pai M. Tuberculosis among health-care workers in low- and middle-income countries: a systematic review. Plos Med 2006; 3 : 2376-91

5.- Menzies D, Fanning A, Yuan L, Fiztgerald M. Tuberculosis among health care workers. N Engl J Med 1995; 332: 92-8.

6.- Bonifacio N, Saito M, Gilman R H, Leung F, Córdova-Chávez N, Chacaltana-Huarcaya J, et al. High risk for tuberculosis in hospital physicians. Emerg Infect Dis 2002; 8: 747-8.

7.- Yanai $\mathrm{H}$, Limpakarnjanarat $\mathrm{K}$, Uthaivoravit $\mathrm{W}$, Mastro T D, Mori T, Tappero J W. Risk of Mycobacterium tuberculosis infection and disease among health care workers, Chiang Rai, Thailand. Int J Tuberc Lung Dis 2003; 7: $36-45$.

8.- Laniado-Laborín R, Cabrales-Vargas N. Tuberculosis in healthcare workers at a general hospital in Mexico. Infect Control Hosp Epidemiol 2006; 27: 449-52.

9.- Silva V M C, Cunha A J L A, Kritski A L. Tuberculin skin test conversion among medical students at a teaching hospital in Rio de Janeiro, Brazil. Infect Control Hosp Epidemiol 2002; 23: 591-4.

10.- Roth V R, Garrett D O, Laserson K F, Starling C E, Kritski A L, Medeiros E A S A multicenter evaluation of tuberculin skin test positivity and conversion among health care workers in Brazilian hospitals. Int J Tuberc Lung Dis 2005; 9: 1335-42.

11.- McKenna M T, Hutton M, Cauthen G, Onorato I M. The association between occupation and tuberculosis. A populationbased survey. Am J Respir Crit Care Med 1996; 154: 587-93.

12.- Díaz-Castrillo A O, Dueñas-Mojena D, Lazo-Álvarez M A, Borroto-Gutiérrez S, González-Ochoa E. Tuberculosis en trabajadores de salud del Hospital Psiquiátrico de la Habana, 1997-2003. Rev Panam Infectol 2005; 7: 22-6.

13.- Naidoo S, Jinabhai C C. TB in health care workers in KwaZulu-Natal, South Africa. Int J Lung Dis 2006; 10: 676-82.

14.- Dimitrova B, Hutchings A, Atun R, Drobniewski F, Marchenko G, Zakharova S, et al. Increased risk of tuberculosis among health care workers in Samara Oblast, Russia: analysis of notification data. Int $\mathrm{J}$ Tuberc Lung Dis 2005; 9: 43-8.

15.- Templeton G L, Illing L A, Young L, Cave D, Stead W W, Bates J H. The risk of transmission of Mycobacterium tuberculosis at the bedside and during autopsy. Ann Intern Med 1995; 122: 955-6.

16.- Kantor H S, Pobrete R, Pusateri S L. Nosocomial transmission of tuberculosis from unsuspected disease. Am J Med 1988; 84: 833-8.

17.- Flavin R J, Gibbons N, O'Briain D S. Mycobacterium tuberculosis at autopsy-exposure and protection: an old adversary revisited. J Clin Pathol 2007; 60: 487-91.

18. - Alonso-Echanove J, Granich R M, Laszlo A, Chu G, Borja N, Blas R, et al. Occupational transmission of Mycobacterium tuberculosis to health care workers in a university hospital in Lima, Peru. Clin Infect Dis 2001; 33: 589-96.
19.- Tipple M A, Heirendt W, Metchock B, Ijaz K, McElroy P D, Andre A M. Tuberculosis outbreak in a community hospitalDistrict of Columbia, 2002. MMWR Morb Mortal Wkly Rep 2004; 53: 214-6.

20.- Greenaway C, Menzies D, Fanning A, Grewal G, Yuan L, FitzGerald J M, et al Delay in diagnosis among hospitalized patients with active tuberculosis. Predictors and outcomes. Am J Respir Crit Care Med 2002; 165: 927-33.

21.- Maloney S A, Pearson M L, Gordon M T, Del Castillo R, Boyle J F, Jarvis W R. Efficacy of control measures in preventing nosocomial transmission of multidrugresistant tuberculosis to patients and health care workers. Ann Intern Med 1995; 122: 90-5.

22.- Valenzuela P. Resistencia y multiresistencia a fármacos antituberculosos en Chile. Rev Chil Salud Pública 2000; 4: 139-44.

23.- Riley R L, Nardell E A. Clearing the air. The theory and application of ultraviolet air disinfection. Am Rev Respir Dis 1989; 139: 1286-94

24.- Menzies D, Fanning A, Yuan L, Fitzgerald M, Canadian Collaborative Group in Nosocomial Transmission of TB. Ann Intern Med 2000; 133: 779-89.

25.- Jereb J A, Klevens R M, Privett T D, Smith P J, Crawford J T, Sharp V L, et al. Tuberculosis in health care workers at a hospital with an outbreak of multidrugresistant Mycobacterium tuberculosis. Arch Intern Med 1995; 155: 854-9.

26.- Sepkowitz K A, Eisenberg L. Occupational deaths among healthcare workers. Emerg Infect Dis 2005; 11: 1003-8.

27. - Ho T B L, Raymer C F J, Lindfield T, Young Y, Whitfield R J. Prevalence of TB in healthcare workers in south west London. Thorax 2004; 59: 1002-3. 
28. - Pai M, Kalantri S, Nath Aggarwal A, Menzies D, Blumberg HM. Nosocomial tuberculosis in India. Emerg Infect Dis 2006; 12: $1311-8$

29. - Centers for Disease Control and Prevention. Guidelines for preventing the transmission of Mycobacterium tuberculosis in healthcare facilities. MMWR Morb Mortal Wkly Rep 2005; 54 (RR17):1-141

30. - Valenzuela M T, Lagos A, Schroeder F, O'Ryan L. Transmisión de la tuberculosis al personal de salud. Rev Chil Infect 1998; 15: 280-4.

31. - De Vries G, Sebek M M, Lambregts-van Weezenbeek C S. Healthcare workers with tuberculosis infected during work. Eur Respir J 2006; 28:1216-21.

32.- Ong A, Rudoy I, González L C, Creasman J, Kawamura L M, Daley C L. Tuberculosis in healthcare workers: A molecular epidemiologic study in San Francisco. Infect Control Hosp Epidemiol 2006; 27:453-8.

33. - Bhanu N V, Banavalikar J N, Kapoor S K, Seth P. Suspected small-scale interpersonal transmission of Mycobacterium tuberculosis in wards of an urban hospital in Delhi, India. Am J Trop Med Hyg 2004; 70: 527-31.

34. - Casas X, Ruiz J, Casas I. Tuberculosis as an occupational disease. Arch Bronchoneumol 2005; 41: 590

35.- Gopinath K G, Siddique S, Kirubakaran H, Shanmugam A, Mathai E, Chandy G M Tuberculosis among healthcare workers in a tertiary-care hospital in South India. J Hosp Infect 2004; 57: 339-42

36. - Moore D A, Evans C A, Gilman R H, Caviedes L, Coronel J, Vivar A, et al. Microscopic-observation drug-susceptibility assay for the diagnosis of TB. N Engl J Med 2006; 355: 15: 1539-50

37. - Frenzel E C, Thomas G A, Hanna H A. The importance of two-step tuberculin skin testing of newly employed health care workers. Infect Control Hosp Epidemiol 2006; 27: 512-24.

38.- Menzies D. Interpretation of repeated tuberculin tests. Boosting, conversion, and reversion. Am J Respir Crit Care Med 1999; 159:15-21

39. - Lee E, Holzman R S. Evolution and current use of the tuberculin test. Clin Infect Dis 2002; 34: 365-70.

40.- Richeldi L. An update on the diagnosis of tuberculosis infection. Am J Respir Crit Care Med 2006; 174: 736-42.

41. - Dheda K, Udwadia Z F, Huggett J F,
Johnson M A, Rook G A. Utility of the antigen-specific-interferon- $\gamma$ assay for the management of tuberculosis. Curr Opin Pulm Med 2005; 11: 195-202.

42. - Pai M, Joshi R, Dogra S, Mendiratta D K, Narang P, Kalantri S, et al. Serial testing of health care workers for tuberculosis using interferon- $\gamma$ assay. Am J Respir Crit Care Med 2006; 174: 349-55.

43. - Mori T, Sakatani M, Yamagishi F, Takashima T, Kawabe Y, Nagao K, et al. Specific detection of tuberculosis infection An interferon- $\gamma$-based assay using new antigens. Am J Respir Crit Care Med 2004; 170: 59-64.

44. - Kang Y A, Lee H W, Yoon H H, Cho B, Han S K, Shim Y S, et al. Discrepancy between the tuberculin skin test and the whole-blood interferon $\gamma$ assay for the diagnosis of latent tuberculosis infection in an intermediate tuberculosis-burden country. JAMA 2005; 293: 2756-61.

45. - Fennelly K P, Nardell E A. The relative efficacy of respirators and room ventilation in preventing occupational tuberculosis. Infect Control Hosp Epidemiol 1998; 19: 754-9.

46. - Harries A D, Maher D, Nunn P. Practical and affordable measures for the protection of healthcare workers from tuberculosis in low-income countries. Bull World Health Organ 1997; 75: 477-89. Review.

47. - Baussano I, Bugiani M, Carosso A, Mairano D, Barocelli A P, Tgana M, et al Risk of tuberculin conversion among healthcare workers and the adoption of preventive measures. Occup Environ Med 2007; 64: 161-6.

48. - Bangsberg D R, Crowley K, Moss A, Dobkin J F, McGregor C, Neu H C. Reduction in tuberculin skin-test conversions among medical house staff associated with improved tuberculosis infection control practices. Infect Control Hospital Epidemiol 1997; 18: 566-70

49. - Blumberg H M, Sotir M, Erwin M, Bachman R, Shulman J A. Risk of house staff tuberculin skin test conversion in an area with a high incidence of tuberculosis. Clin Infect Dis 1998; 27: 826-33.

50. - Wenger P N, Otten J, Breeden A, Orfas D, Beck-Sague C M, Jarvis W R. Control of nosocomial transmission of multidrugresistant Mycobacterium tuberculosis among healthcare workers and HIV-infected patients. Lancet 1995; 345: 235-40
51.- Harries A D, Hargreaves N J, Gausi F, Kwanjana J H, Salaniponi F M. Preventing tuberculosis among health workers in Malawi. Bull World Health Organ 2002; 80: 526-31.

52.- Escombe A R, Oeser C C, Gilman R H, Navincopa M, Ticona E, Pan W, et al Natural ventilation for the prevention of airborne contagion. Plos Med 2007; 4: 309-17.

53. - Marcus A M, Rose D N, Sacks H S, Schetcher C B. BCG vaccination to prevent tuberculosis in health care workers: A decision analysis. Prev Med 1997; 26: 201-7.

54.- Nettleman M, Geerdes H, Roy M C. The cost-effectiveness of preventing tuberculosis in physicians using tuberculin skin testing or a hypothetical vaccine. Arch Intern Med 1997; 157: 1121-7

55.- Zúñiga M, Valenzuela $P$, Farga V, Yáñez A, Rojas M (Comité Editorial). Programa Nacional de Control de la Tuberculosis. Norma General Técnica $N^{\circ}$ 82. Subsecretaría de Salud Pública y División de Prevención y Control de Enfermedades. Ministerio de Salud de Chile, 2005

56.- Erzo A. Recomendaciones y actualización de la normativa de aislamiento de pacientes del Programa de Infecciones Intrahospitalarias. Ministerio de Salud de Chile. Circular No 26 , septiembre, 1998

57.- Karonga Prevention Trial Group Randomized controlled trial of single $\mathrm{BCG}$, repeated $\mathrm{BCG}$, or combined $\mathrm{BCG}$ and killed Mycobacterium leprae vaccine for prevention of leprosy and tuberculosis in Malawi. Lancet 1996; 348: 17-24.

58. - Broekmans J F, Migliore G B, Rieder H L, Lees J, Ruutu P, Loddenkemper R, et al. European framework for tuberculosis control and elimination in countries with low incidence. Eur Resp J 2002; 19 : 765-75.

59. - National Institute for Health and Clinical Excellence. Tuberculosis: clinical diagnosis and management, and measures for its prevention and control. London; March 2006. (Disponible en: http:// www.nice.org.uk/nicemedia/pdf/ CG033niceguideline.pdf)

60.- Humphreys H. Control and prevention of healthcare-associated tuberculosis: the role of respiratory isolation and personal respiratory protection. J Hosp Infect 2007; 66: $1-5$. 